\title{
Cross-Phase Modulation Induced by OOK Channels on Higher-Rate DQPSK and Coherent QPSK Channels
}

\author{
Alberto Bononi, Marco Bertolini, Graduate Student Member, IEEE, Paolo Serena, and Giovanni Bellotti
}

\begin{abstract}
In this paper we show that, in hybrid wavelength division multiplexed systems, the performance of high datatrate QPSK channels impaired by cross-phase modulation (XPM) induced by the lower rate OOK channels can be simply estimated by an extension of a well-known linear model for XPM, and novel analytical expressions of the sensitivity penalty are provided. From such a model we prove that the reported QPSK penalty decrease with QPSK baudrate increase should be attributed to the action of the phase estimation process rather than to the walkoff effect. The model also simply shows how coherent QPSK is more affected by XPM than incoherent DQPSK, and allows to infer that even more impact is expected when the baudrate is further reduced through polarization multiplexing.
\end{abstract}

Index Terms-Cross phase modulation (XPM), differential quadrature phase shift keying (DQPSK), phase estimation, phase shift keying.

\section{INTRODUCTION}

I N ORDER TO make the deployment of 40 and $100 \mathrm{~Gb} / \mathrm{s}$ services in optical terrestrial networks cost effective, high datarate channels must be loaded on an already existing wavelength-division multiplexing (WDM) infrastructure designed for $10 \mathrm{~Gb} / \mathrm{s}$ on-off keying (OOK) channels with a channel spacing down to $50 \mathrm{GHz}$. The modulation format employed in the upgraded channels must thus feature both high spectral efficiency and good tolerance to narrow optical filtering. Among the proposed modulation formats that meet those requirements, quadrature phase shift keying (QPSK) has been investigated, both using a standard incoherent receiver and differential phase encoding (DQPSK) [1], and with coherent reception and feedforward phase estimation [2], [3].

The main limitation of QPSK operated in a hybrid scenario, i.e., with two or more different formats mixed on the WDM comb, lies in its limited tolerance to cross phase modulation (XPM) caused by neighboring intensity-modulated OOK channels. Both simulation and experiments [4]-[8] have already established that this is the main impairment on QPSK channels in

Manuscript received December 02, 2008; revised March 02, 2009. First published April 28, 2009; current version published August 21, 2009.

A. Bononi, M. Bertolini and P. Serena are with the Dipartimento di Ingegneria dell'Informazione, Università degli Studi di Parma, 43100 Parma, Italy (e-mail: bononi@tlc.unipr.it; bertolini@tlc.unipr.it; serena@tlc.unipr.it).

G. Bellotti is with Alcatel-Lucent, Optics Division, 20059 Vimercate, Italy (e-mail: giovanni.bellotti@alcatel-lucent.it).

Color versions of one or more of the figures in this paper are available online at http://ieeexplore.ieee.org.

Digital Object Identifier 10.1109/JLT.2009.2021537
QPSK/OOK hybrid systems. Two analytical models have been proposed for such hybrid systems to estimate the penalty on DPSK and DQPSK channels. A first model [9] builds on previous work of Ho on DPSK [10] and is based on explicit formulas of the probability density function (PDF) of the received phase. A second model [11], [12] is based instead on explicit formulas of the PDF of the electrical current, and works only when the intensity fluctuations caused by XPM are dominant.

Recent work by Vassilieva [13] also showed that the impact of XPM is reduced when the baudrate of the upgraded DQPSK channels is increased, and the reason was attributed to the increased OOK bit walkoff seen by the DQPSK channels when their symbol time gets reduced.

In this paper, a simple theoretical framework is presented that models the interactions among OOK and QPSK channels through XPM. The framework is an extension of the work in [9]. The main novelties are: i) a rigorous estimation of XPM in a dispersion managed (DM) setting, including pre-compensation, post-compensation and in-line compensation, and a new theoretical formulation that makes it possible to simply extend the calculations to any DM link with arbitrary dispersion/power profile; ii) simple analytical fits of the sensitivity penalty versus phase offset variance for both incoherent and coherent reception; iii) the extension of results to the coherent QPSK format with feed-forward phase estimation; and iv) a thorough verification of the theoretical sensitivity penalty against Split-Step Fourier simulations.

Using this approach, we are able to thoroughly and simply explain the decrease of the penalty with the increase in datarate of the QPSK channels, both with differential and with coherent reception, and we prove that it is mostly due to the reduction of the XPM operated by the phase estimation, rather than by the channel walkoff. The impact of XPM on the number of symbols involved in the feedforward phase estimation for coherent QPSK is also highlighted. The proposed framework is very general and can be used as a guide to the design of hybrid systems, since novel, very simple formulas are provided to calculate the XPM induced sensitivity penalty for both DQPSK and coherent QPSK formats.

The paper is organized as follows: in Section II we derive analytical approximations for the sensitivity penalty of DQPSK/QPSK channels impaired by XPM-induced phase noise. In Section III we extend a well known small-signal model to predict the XPM induced phase variance. In Section IV we test our analytical results against numerical simulations and finally in Section V we draw our conclusions. 


\section{BER WITH PHASE NOISE}

In this section we will start from standard results on the BER evaluation for PSK modulated signals in the presence of both additive noise and a phase offset [14], and tailor them to find the BER of our upgraded optical (D)QPSK channels with XPM induced phase noise, similarly to the work in [15], [16]. In our optical system, the received optical field $\tilde{E}(t)$ is the sum of a propagation-distorted signal component $\tilde{A}(t)$ and amplified spontaneous emission (ASE) noise $\tilde{m}(t)$ cumulated along the dispersion-managed line. In normal operating conditions, the XPM far exceeds the ASE-induced nonlinear phase noise [17] on the QPSK channels and is by far the dominant impairment [6]-[8]. Moreover, as explained in the Appendix I, thanks to the effect of walk-off, the XPM tends to have Gaussian statistics by the central limit theorem, since it is the sum of the contributions of many different symbols of the interfering signals.

In the case of DQPSK modulation, the receiver consists of an optical filter followed by a delay interferometer and balanced photo-detection [16]. As shown in the Appendix II, the BER of a DQPSK signal affected by both additive circular Gaussian noise $\tilde{m}(t)$ and the XPM Gaussian phase noise process $\Delta \phi$, and received by an interferometric receiver, can be expressed as $^{1}[10]$

$$
\begin{aligned}
\mathrm{BER}=\frac{3}{8}-\frac{\rho}{4} e^{-\rho} \sum_{n=1}^{\infty}\left[I_{\frac{n-1}{2}}\left(\frac{\rho}{2}\right)\right. & \left.+I_{\frac{n+1}{2}}\left(\frac{\rho}{2}\right)\right]^{2} \\
& \times \frac{\sin \left(n \frac{\pi}{4}\right)}{n} e^{-\frac{\operatorname{Var}[\Delta \phi]}{2} n^{2}}
\end{aligned}
$$

where $I_{\alpha}(x)$ is the modified Bessel function of fractional index $\alpha$, and $\rho$ is the signal to noise ratio (SNR), with the noise variance evaluated over the one-sided bandwidth of the optical filter. From (1) we find the following novel best-fit of the sensitivity penalty (SP) at a given reference back-to-back BER:

$$
S P \cong-8.5 \log _{10}\left(1-\rho_{\text {ref }} \operatorname{Var}[\Delta \phi(t)]\right)
$$

where $\rho_{\text {ref }}$ is the SNR that achieves the reference BER, namely $\rho_{\text {ref }}=61.7$ at BER $=10^{-9}$, and $\rho_{\text {ref }}=31.4$ at BER $=$ $10^{-5}$.

In the case of coherent reception, for simplicity we assume the local oscillator is aligned with the polarization of the incoming signal, and the optical filter bandwidth is the same as for the DQPSK receiver. We assume the frequency of the local oscillator is matched to that of the incoming signal (homodyne case), and the feedforward phase estimation error is still denoted as $\Delta \phi$. Since in this case no additive optical noise is present on the local oscillator field, the BER of the QPSK channel affected by a Gaussian phase error $\Delta \phi$ can be computed, similarly to the DQPSK case and as shown in the Appendix II, as

$$
\begin{array}{r}
\mathrm{BER}=\frac{3}{8}-\frac{1}{2} \sqrt{\frac{\rho}{\pi}} e^{-\rho / 2} \sum_{n=1}^{\infty}\left[I_{\frac{n-1}{2}}\left(\frac{\rho}{2}\right)+I_{\frac{n+1}{2}}\left(\frac{\rho}{2}\right)\right] \\
\times \frac{\sin \left(n \frac{\pi}{4}\right)}{n} e^{-\frac{\operatorname{Var}[\Delta \phi]}{2} n^{2}} .
\end{array}
$$

${ }^{1}$ Note that the BER formula in [9] includes also nonlinear phase noise, which we neglected here, but incorrectly uses $e^{-\operatorname{Var}[\Delta \phi] n^{2}}$ for the XPM contribution. This is ironic, since such a reference correctly reports an important typo in the seminal paper by Ho.

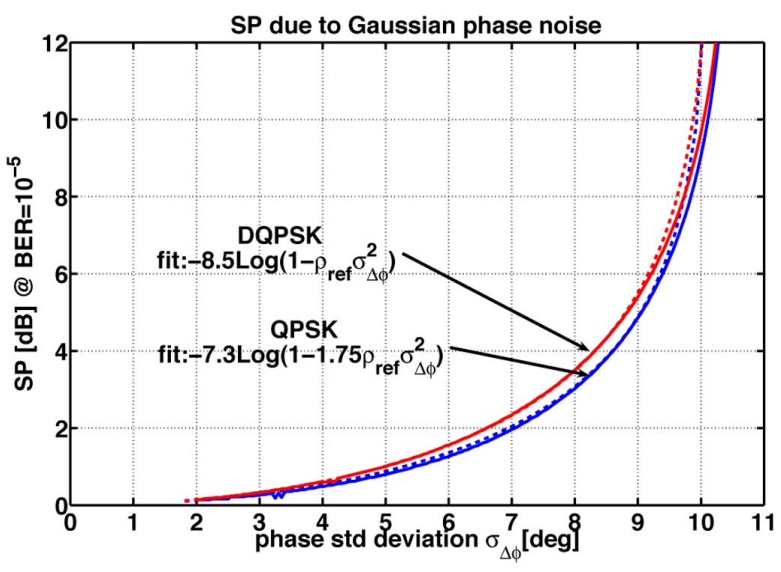

Fig. 1. Sensitivity penalty at $\mathrm{BER}=10^{-5}$ versus standard deviation of phase error $\sigma_{\Delta \phi}=\sqrt{\operatorname{Var}[\Delta \phi]}$. Solid lines: "Blachman" formulas (1) for DQPSK and (3) for coherent QPSK. Dashed lines: best fits (2) and (4).

Similarly to the DQPSK case, we obtain the following novel best-fit of the SP obtained from (3) at a reference back-to-back BER:

$$
S P \cong-7.3 \log _{10}\left(1-1.75 \rho_{\text {ref }} \operatorname{Var}[\Delta \phi(t)]\right)
$$

where for QPSK we have $\rho_{\text {ref }}=36.0$ at BER $=10^{-9}$, and $\rho_{\text {ref }}=18.1$ at BER $=10^{-5}$. The goodness of fit of (2) and (4) is shown in Fig. 1 at a reference BER $=10^{-5}$. The fit both at $\mathrm{BER}=10^{-3}$ and at $\mathrm{BER}=10^{-9}$, not reported, is essentially as good as here.

In order to effectively use (2) and (4), we need to calculate the variance of the phase error induced by XPM, which can be computed as $\operatorname{Var}[\Delta \phi(t)]=\int_{-\infty}^{\infty} C_{\Delta \phi}(f) \mathrm{d} f$, where $C_{\Delta \phi}(f) \triangleq \mathcal{F}\left\{E[\Delta \phi(t-\tau) \Delta \phi(t)]-E[\Delta \phi(t)]^{2}\right\}$ is the power spectral density (PSD), i.e., the Fourier transform $(\mathcal{F})$ of the autocovariance of $\Delta \phi(t)$. We will describe a method to estimate $C_{\Delta \phi}(f)$ in the following sections.

\section{IM-XPM FILTER}

In this section we will extend a well-established small-signal model for estimating the intensity-modulation (IM) to XPM conversion in DM WDM optical links [18], [19]. Our extended model includes the effect of pre-, post-, and in-line compensating fibers, and does take into account the GVD-induced pump distortion, along the lines of the "improved" IM-XPM-IM filters described in [20]-[22] and well suited for $40 \mathrm{~Gb} / \mathrm{s}$ OOK transmissions, whose simplified version, applicable to $10 \mathrm{~Gb} / \mathrm{s}$ OOK systems, was first published in [23]-[25] and later experimentally verified in [26]. We will then use the obtained results to calculate the variance induced on the phase of DQPSK/QPSK signals by neighboring OOK channels. Following the mathematical derivation in Appendix III, we can relate the Fourier transform of the XPM of the received reference channel $s$, $\Theta_{\mathrm{XPM}}(\omega)$, to the Fourier transform of the input power $P_{p}(0, \omega)$ of "pump" channel $p$ as: $\Theta_{\mathrm{XPM}}(\omega)=H_{\mathrm{XPM}, p}(\omega) P_{p}(0, \omega)$, where the IM-XPM filter has expression

$$
H_{\mathrm{XPM}, p}(\omega)=-\frac{\Phi_{N L}}{2 P_{\mathrm{in}, \mathrm{p}}}\left\{e^{-j \frac{\omega^{2}}{2} \beta_{r}} H_{p}(\omega)+e^{j \frac{\omega^{2}}{2} \beta_{r}} H_{p}^{*}(-\omega)\right\}
$$


where $\Phi_{N L}$ is the average cumulated nonlinear phase of the pump channel, $P_{\mathrm{in}, \mathrm{p}}$ is the pump average input power, $\beta_{r}\left[\mathrm{ps}^{2}\right]$ is the dispersion accumulated over the entire DM link (including pre-, in-line and post-compensation) and $H_{p}(\omega)$ is a filter whose expression is given in the Appendix III. This same result can be obtained using a general approach based on a regular perturbation of the dispersion-managed nonlinear Schroedinger equation [27]. Most importantly, in [27] it is shown that the filter $H_{p}(\omega)$ in (5) is a simple function of the so called DM link kernel, a quantity that determines all performance metrics of the DM link. Hence, when moving to a DM system with a different dispersion and/or power profile, it is enough to update the expression of the kernel and thus of filter $H_{p}$, without having to directly recompute the IM-XPM filter.

Note that only the simplest case of such IM-XPM filter, namely the case of full in-line compensation, was used in [9] using the approximate filter in [10].

Also, note that the presented model can be extended to take into account even the effect of SPM on both pump and probe, along the lines of the method developed in [28].

To verify the accuracy of the analytical filter, we compared it to the "true" filter, obtained from simulations using the split-step Fourier method (SSFM) as follows. We sent two signals along a DM system, composed of a linear pre-compensating fiber, $N=15$ identical spans with linear dispersion compensation at the end of each span, and finally a post-compensating fiber. The transmission fiber was a non-zero dispersion shifted fiber (NZDSF) with dispersion $D_{T X}=3.83 \mathrm{ps} /(\mathrm{nm} \mathrm{km})$. The dispersion of the pre-compensating fiber was selected according to the "straight line rule" (SLR) [29], [30] as: $D_{\text {pre }}=-\left(D_{T X} / \alpha\right)-(N-1 / 2) D_{\text {in }}$, where three different values of the residual in-line dispersion per span were used: $D_{\text {in }}=[0,50,100] \mathrm{ps} / \mathrm{nm}$. The post-compensation $D_{\text {post }}$ was such that the total cumulated dispersion $D_{\text {tot }}=D_{\text {pre }}+N D_{\text {in }}+D_{\text {post }}$ was zero. At the line input, the "probe" channel was $\mathrm{CW}$, while the "pump," at a frequency distance $\Delta f=50 \mathrm{GHz}$ from probe, was sinusoidally modulated in power as $p_{p}(0, t)=P_{\text {in, }}\left(1+m_{p} \cos \left(\omega_{m} t\right)\right)$, with power modulation index $m_{p}=0.9$. The average nonlinear phase cumulated by both channels along the link was $0.3 \pi$. At the receiver, no optical/electrical filtering was added.

In the SSFM, the FFT time window contained $6.4 \mathrm{nsec}$ with 320 time samples/nsec, and the maximum nonlinear phase rotation per space-step was set to $1 \mathrm{mrad}$. We only simulated the effect of XPM, i.e., we turned off self-phase modulation (SPM) and four-wave mixing (FWM). From the Fourier transform of the transmitted power of pump channel $P_{p}(0, f)$ and that of the received probe phase $\Theta_{\mathrm{XPM}}(f)$, we numerically computed the IM-XPM filter as $H_{\mathrm{XPM}, p}\left(f_{m}\right)=\Theta_{\mathrm{XPM}}\left(f_{m}\right) / P_{p}\left(0, f_{m}\right)$, being $f_{m}$ the sinusoidal modulation frequency. Fig. 2 shows the IM-XPM filter amplitude in $\mathrm{dB}$ for both theory (solid) and simulation (dots). The frequency is normalized to the baudrate of the OOK channels $R_{\mathrm{OOK}}=10$ Gbaud. The plots of the amplitude versus frequency can be interpreted by recalling from the Appendix III that the IM-XPM filter $H_{\mathrm{XPM}, p}$ results from the composition of two factors.

1) A walkoff factor (22) which gives $H_{\mathrm{XPM}, p}$ a typical lowpass behavior, as seen at full in-line compensation $D_{\text {in }}=$ 0 ;

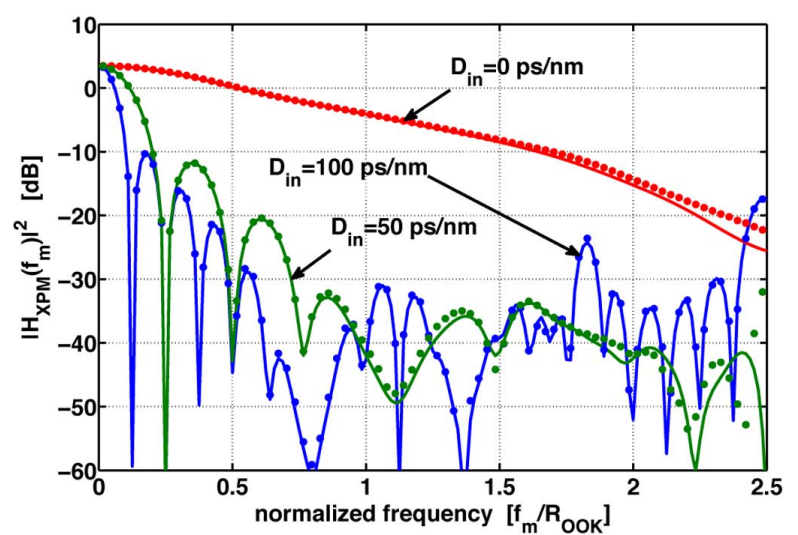

Fig. 2. IM-XPM filter amplitude $10 \log _{10}\left|H_{\mathrm{XPM}, p}\left(f_{m}\right)\right|^{2}$ versus normalized frequency $f_{m} / R_{\mathrm{OOK}}$, with $R_{\mathrm{OOK}}=10 \mathrm{GHz}$. 15-span NZDSF system, $D_{\text {pre }}=\mathrm{SLR}, D_{\text {tot }}=0$ and three values of in-line dispersion per span $D_{\text {in }}=[0,50,100] \mathrm{ps} / \mathrm{nm}$. Nonlinear phase $0.3 \pi$. Pump-probe spacing $\Delta f=50 \mathrm{GHz}$. Dots: SSFM simulated filter; Solid line: analytical filter (5).

2) An interference factor (21), which introduces oscillations in frequency and notches in $\left|H_{\mathrm{XPM}, p}\right|$ whose frequencies decrease for increasing $D_{\text {in }}$, as visible in the figure when the in-line dispersion is non-zero.

From the comparison of simulation and theory we note that the predictions of the analytical filter are in very good agreement with SSFM simulations, but the precision of the filter tends to worsen at higher frequencies.

\section{Phase Variance Evaluation}

Having now an accurate filter that describes how the power fluctuations on a single OOK channel are impressed on the phase of the probe PSK signal, the overall XPM can be written as a superposition of the XPM stemming from the individual OOK channels: $\Theta_{\mathrm{XPM}}(f)=\sum_{\substack{p=-M \\ p \neq 0}}^{M} H_{\mathrm{XPM}, p}(f) P_{p}(0, f)$, having assumed that the probe channel $s=0$ has $M$ OOK pump channels to its right and $M$ to its left. The power spectral density of the XPM process is: $C_{\mathrm{XPM}}(f)=\sum_{\substack{p=-M \\ p \neq 0}}^{M}\left|H_{\mathrm{XPM}, p}(f)\right|^{2} C_{\mathrm{OOK}}(f)$, where we used the independence of the OOK channels, and where $C_{\mathrm{OOK}}(f)$ is the PSD of the intensity of each of the input OOK signals, which, for non-return to zero (NRZ) OOK modulation is

$$
C_{\mathrm{OOK}}(f)=\left(\frac{P_{\mathrm{in}, \mathrm{p}}}{R_{\mathrm{OOK}}} \frac{r-1}{r+1}\right)^{2} \operatorname{sinc}^{2}\left(\frac{f}{R_{\mathrm{OOK}}}\right)
$$

where $r>1$ is the extinction ratio. Now, the PSD $C_{\Delta \phi}(f)$ needed for the variance calculation is related to $C_{\mathrm{XPM}}(f)$ according to the specific demodulator structure as detailed below.

\section{A. $D Q P S K$}

Assume the upgraded channel at a baudrate of $R_{s}=1 / T_{s}$ (Gbaud) is DQPSK modulated. The receiver consists of an optical filter followed by a delay-demodulation interferometer with balanced detection. Hence the phase error to be used in the BER formula (1) for the reference channel $s$ is the difference $\Delta \phi(t)=\theta_{\mathrm{XPM}}(t)-\theta_{\mathrm{XPM}}\left(t-T_{s}\right)$. Taking Fourier transforms one gets: $\Delta \Phi(\omega)=\Theta_{\mathrm{XPM}}(\omega) H_{D}(\omega)$, where the differential 
phase filter is $H_{D}(\omega)=1-e^{-j \omega T_{s}}$, with $\left|H_{D}(\omega)\right|^{2}=$ $4 \sin ^{2}\left(\omega / 2 R_{s}\right)$. Hence $C_{\Delta \phi}(f)=C_{\mathrm{XPM}}(f)\left|H_{D}(f)\right|^{2}$, so that

$$
\operatorname{Var}[\Delta \phi]=\sum_{\substack{p=-M \\ p \neq 0}}^{M} \int_{-\infty}^{\infty} C_{\mathrm{OOK}}(f) \cdot\left|H_{\mathrm{XPM}, p}(f)\right|^{2}\left|H_{D}(f)\right|^{2} \mathrm{~d} f
$$

and we note that the integrand must have Hermitian symmetry, so that the integral is twice that on positive frequencies. Since the optical filter also gives a spectral shaping to the phase [31], it is sufficient to restrict the range of integration to the bandwidth $B_{o}$ of the optical filter [10]. Also, it can be shown that $H_{X P M,-p}(f)=H_{\mathrm{XPM}, p}^{*}(f)$, so that finally the variance expression simplifies to

$$
\operatorname{Var}[\Delta \phi]=2 \sum_{p=1}^{M} 2 \int_{0}^{B_{o}} C_{\mathrm{OOK}}(f)\left|H_{\mathrm{XPM}, p}(f)\right|^{2}\left|H_{D}(f)\right|^{2} \mathrm{~d} f .
$$

Note that, when we can approximate $\omega+p \Delta \omega \cong$ $p \Delta \omega$, then from (23) $H_{p}(\omega)=H_{p}^{*}(-\omega)$ and thus from (5) we get (see Appendix III): $\left|H_{\mathrm{XPM}, p}(\omega)\right|^{2} \cong$ $(N \gamma / \alpha)^{2}\left|I_{p}(\omega p \Delta \omega)\right|^{2}\left|H_{W p}(\omega p \Delta \omega)\right|^{2} \cos \left(\left(\omega^{2} / 2\right) \beta_{r}\right)$ which is the approximate expression used by Ho ([10], (9), where $\beta_{r}=0$ is assumed). Such an approximation, while reasonable at $100 \mathrm{GHz}$ channel spacing, becomes more critical at $50 \mathrm{GHz}$ spacing.

\section{B. Coherent QPSK}

We next assume the reference channel $s$ is QPSK modulated and demodulated using a coherent receiver, with feedforward phase estimation based on the Viterbi and Viterbi (V\&V) algorithm [32]. We also assume the feedforward phase estimation error $\Delta \phi(t)$ is dominated by the XPM rather than by the phase induced by the ASE on the received signal. Namely, the phase error (or "offset") to be used in the BER formula (3) is the difference $\Delta \phi(t)=\theta_{\mathrm{XPM}}(t)-\hat{\theta}_{\mathrm{XPM}}$, where as in [33] we assume the estimated XPM comes from a linear processing of the sampled values at the $K$ previous symbol times [33, Eqs. (15), (27)]

$$
\hat{\theta}_{\mathrm{XPM}}=\frac{\sum_{k=1}^{K} \theta_{\mathrm{XPM}}\left(t-k T_{s}\right)}{K}=\frac{\sum_{k=1}^{K} \arg \left[\tilde{E}\left(t-k T_{s}\right)^{4}\right]}{4 K} .
$$

This is less performant but simpler to analyze than the optimal $\mathrm{V} \& \mathrm{~V}$ phase estimator [34]: $\hat{\theta}=(1 / 4) \arg \left[(1 / K) \sum_{k=1}^{K} \tilde{E}(t-\right.$ $\left.k T_{s}\right)^{4}$. The difference between the two estimators is most striking when the fields $\tilde{E}\left(t-k T_{s}\right)$ have widely different amplitudes and thus SNRs $\rho_{k}$ at the different symbol times. However, when treating penalties stemming from XPM, it is enough to consider the case of equal SNRs, in which case the $\mathrm{V} \& \mathrm{~V}$ and the estimator (8) are quite similar. Using (8), we see that the phase error is a linear filtering of the XPM process: $\Delta \phi(t)=\theta_{\mathrm{XPM}}(t) \otimes h_{D}(t)$, where $\otimes$ denotes convolution, and the filter impulse response is $h_{D}(t)=\delta(t)-(1 / K) \sum_{k=1}^{K} \delta\left(t-k T_{s}\right)$ so that its frequency response is

$$
H_{D}(\omega)=1-\frac{1}{K} \sum_{k=1}^{K} e^{-j \omega k T_{s}}
$$

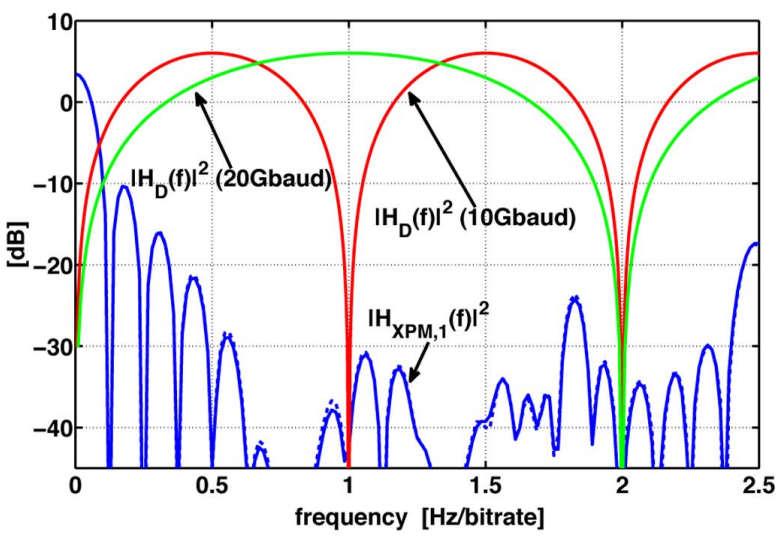

Fig. 3. Plot of $\left|H_{\mathrm{XPM}, 1}(f)\right|^{2}$ and $\left|H_{D}(f)\right|^{2}$ versus normalized frequency $f / R_{\mathrm{OOK}}$, with $R_{\mathrm{OOK}}=10 \mathrm{GHz}$, for DQPSK at 10 and 20 Gbaud. 15-span NZDSF system, $D_{\text {in }}=100 \mathrm{ps} / \mathrm{nm}, D_{\text {pre }}=\mathrm{SLR}, D_{\text {tot }}=0$. Nonlinear phase $0.3 \pi$. Channel spacing $\Delta f=50 \mathrm{GHz}$.

When $K=1$ we get the same differential filter as in the DQPSK case with delay demodulation treated in the previous section. Using expression (9) for what we call the "generalized" differential filter, the variance of the phase error is still given by (7).

\section{Impact of $H_{D}(\omega)$}

The dependence of XPM on the DM link parameters comes through the IM-XPM filter (20). However, as we can see from (7), the variance of XPM also depends on the phase estimation filter $H_{D}(\omega)$. For the same 15-span DM line as in the previous section with $D_{\text {in }}=100 \mathrm{ps} / \mathrm{nm}$ with minimum channel spacing of $50 \mathrm{GHz}$, Fig. 3 depicts both $\left|H_{\mathrm{XPM}, p}(\omega)\right|^{2}$ with $p=1$ (pump-probe spacing $50 \mathrm{GHz}$ ), and $\left|H_{D}(\omega)\right|^{2}$ for a DQPSK reference channel modulated at both $10 \mathrm{Gbaud}(20 \mathrm{~Gb} / \mathrm{s})$ and $20 \mathrm{Gbaud}(40 \mathrm{~Gb} / \mathrm{s})$. The frequency axis is normalized to the bitrate of the pump OOK channel.

It is clear that if the baudrate of the DQPSK channel increases, $H_{D}(\omega)$ gets more effective in suppressing the low frequencies of the XPM spectrum $C_{\mathrm{OOK}}(f)\left|H_{\mathrm{XPM}, p}(f)\right|^{2}$. This result can also be easily understood by reasoning in the time domain: if DQPSK and OOK have the same baudrate, the XPM contributions on adjacent symbols are almost independent and the effect of differential detection cannot help. On the other hand, if the baudrate of DQPSK increases, every OOK symbol induces the same XPM over more and more DQPSK symbols, which are thus correlated. In this case differential detection can partially suppress the XPM contributions.

Fig. 4 shows $\left|H_{D}(\omega)\right|^{2}$ for coherent QPSK at a baudrate of 20 Gbaud, when the phase is estimated over $K=1,3$ or 5 symbols. Increasing $K$ has the effect of reducing the cutoff frequency of $H_{D}(\omega)$, thus increasing the XPM variance. It is a known fact that in absence of strong nonlinearities and when ASE noise dominates over the phase noise of the transmitting laser, a higher $K$ yields a better performance [33]. In the nonlinear regime, on the contrary, $K$ must be reduced to improve the performance [8]. This behavior is the effect of a tradeoff of the filtering action of $H_{D}(\omega)$ on both the ASE- and the XPM-induced estimated phase noise spectrum. If the spectrum of the ASE-induced estimated phase noise (which we overlooked in the above analysis) is almost flat on the bandwidth of the signal, 


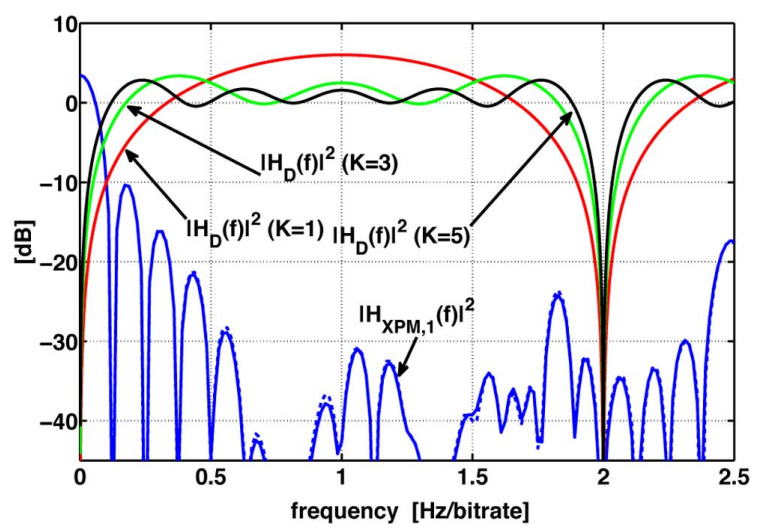

Fig. 4. Plot of $\left|H_{\mathrm{XPM}, 1}(f)\right|^{2}$ and $\left|H_{D}(f)\right|^{2}$ for coherent QPSK at $20 \mathrm{Gbaud}$, varying the number of phase estimation samples $K$. 15-span NZDSF system, $D_{\text {in }}=100 \mathrm{ps} / \mathrm{nm}, D_{\text {pre }}=\mathrm{SLR}, D_{\text {tot }}=0$. Nonlinear phase $0.3 \pi$. Channel spacing $\Delta f=50 \mathrm{GHz}$.

the larger $K$ is, the smaller is the phase error variance. On the other hand, if the phase estimation error has a lowpass spectrum (such as the one induced by XPM or by the lasers phase noise [33]), the smaller $K$ is, the smaller is the variance.

The presented approach can be useful to derive simple rules for the design of the phase estimation stage of coherent receivers. Also note that if the XPM is by far the dominant impairment, the standard differential demodulation of DQPSK is optimal. This is in good agreement with the results in [35].

\section{Checks Against Simulation}

\section{A. Phase Variance Estimation}

To verify the accuracy and the limits of the XPM variance formula (7), we ran Monte Carlo simulations to directly estimate the variance of the received phase. We tested a WDM system composed of a central NRZ QPSK channel at a variable baudrate $10 \leq R_{s} \leq 50$, having $M=2$ NRZ-OOK $10 \mathrm{~Gb} / \mathrm{s}$ channels on each side (5 channels total), with a minimum frequency spacing $\Delta f$ of either 50 or $100 \mathrm{GHz}$. The line was composed of $N=15$ identical spans of $100 \mathrm{~km}$ of either NZDSF fiber $\left(D_{T X}=3.83 \mathrm{ps} / \mathrm{nm}, \alpha=0.22 \mathrm{~dB} / \mathrm{km}\right.$, $\gamma=1.5(1 / W \cdot \mathrm{km}) @ 1550 \mathrm{~nm}$ ) or single mode fiber (SMF, $D_{T X}=17 \mathrm{ps} / \mathrm{nm}, \alpha=0.22 \mathrm{~dB} / \mathrm{km}, \gamma=1.4(1 / W \cdot \mathrm{km}) @$ $1550 \mathrm{~nm})$ and the compensating fibers were linear. The in-line residual dispersion per span was set to $D_{\text {in }}=100 \mathrm{ps} / \mathrm{nm}$, and the pre-compensation was selected using the SLR. The total dispersion $D_{\text {tot }}$ was set to zero, i.e., to the optimal value for PSK, by tuning the post-compensation. Note that for non-zero values of $D_{\text {tot }}$ also the effect of the intensity noise caused by XPM should be taken into account [11], [12].

In the SSFM simulations, we solved the coupled propagation equations for each channel in the single-polarization case, neglecting the FWM beat terms. This amounts to assuming co-polarized WDM channels, which is a worst case for XPM, and neglecting polarization effects. Another implication of our SSFM method is that simulated results for large baudrates $R_{s}$ such that $R_{s}+R_{\mathrm{OOK}}$ exceeds the channel spacing $\Delta f$ neglect the effect of the spectral overlap of the PSK channel with the neighboring OOK channels during propagation [13]. The number of simulated symbols in the FFT window was fixed to 1260 for the QPSK channel, with 60 samples

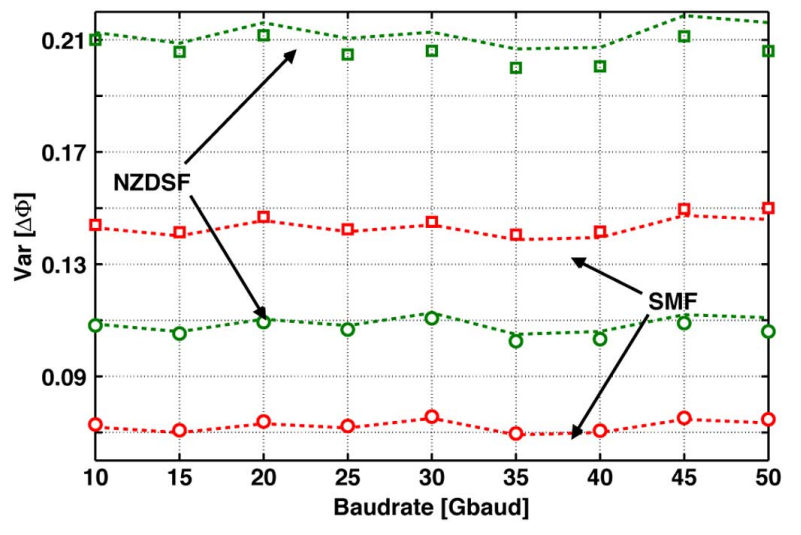

Fig. 5. XPM-induced phase variance on the reference (D)QPSK channel before $H_{D}(\omega)$ in a 15-span 5-channel hybrid DM system with either NZDSF or SMF transmission fiber, $D_{\text {in }}=100 \mathrm{ps} / \mathrm{nm}, D_{\text {pre }}=\mathrm{SLR}, D_{\text {tot }}=0$. SSFM simulation (symbols) and XPM filter prediction (dashed). Channel spacing: $\Delta f=$ $50 \mathrm{GHz}$ (squares); $\Delta f=100 \mathrm{GHz}$ (circles).

per symbol. Such a number was chosen such that the ratios $R_{s} / R_{\mathrm{OOK}}=[1,1.5,2,2.5,3,3.5,4,4.5,5]$ give an integer number of OOK bits within the FFT window. The minimum number of OOK symbols is thus 252, when the QPSK baudrate is 50 Gbaud.

The single-polarization receiver was either an incoherent differential receiver or a coherent receiver with feedforward phase estimation, both with an optical filter with one-sided bandwidth $B_{o}=2 R_{S}$ and no electrical filter.

In all the Monte Carlo simulations, we evaluated the phase variance of every symbol in the sequence separately, taking only the central sample, and subtracting the phase associated with the transmitted symbol. The propagation was repeated 50 times, each time changing the random delay and the random pattern of the OOK channels. We estimated the variance separately on each symbol because, even after subtracting the transmitted phase, the average received phase was different from symbol to symbol, since the mean nonlinear phase induced by XPM depends on the transmitted patterns. After measuring the variance separately on every symbol, we computed the mean of the collected variances. We verified that our procedure produces essentially the same results as the more intuitive procedure in which the phase variance from all the symbols in one simulation is evaluated, and then the average of the phase variance from all the repeated simulations is taken; however our procedure has a faster convergence to the desired confidence level.

We first measured the phase variance of the received reference channel after the optical filter but before demodulation, where the QPSK and DQPSK signals are identical. We compared the SSFM-simulated variance with the theoretical results of (7) when setting $H_{D}(\omega) \equiv 1$. In this case, $C_{\mathrm{OOK}}(f)$ was estimated from the actual OOK waveforms by taking the average of the sample PSDs (i.e., their periodograms) collected during the SSFM simulation. This is because, for short to moderate length bit sequences, $C_{\mathrm{OOK}}(f)$ may have non-negligible variations from its ergodic limit (6).

The results are reported in Fig. 5 for the case of both NZDSF and SMF transmission fiber, using either $50 \mathrm{GHz}$ or $100 \mathrm{GHz}$ channel spacing. The launched average power for all channels was $P_{\text {in }}=2 \mathrm{dBm}$ in each case, corresponding to a cumulated 

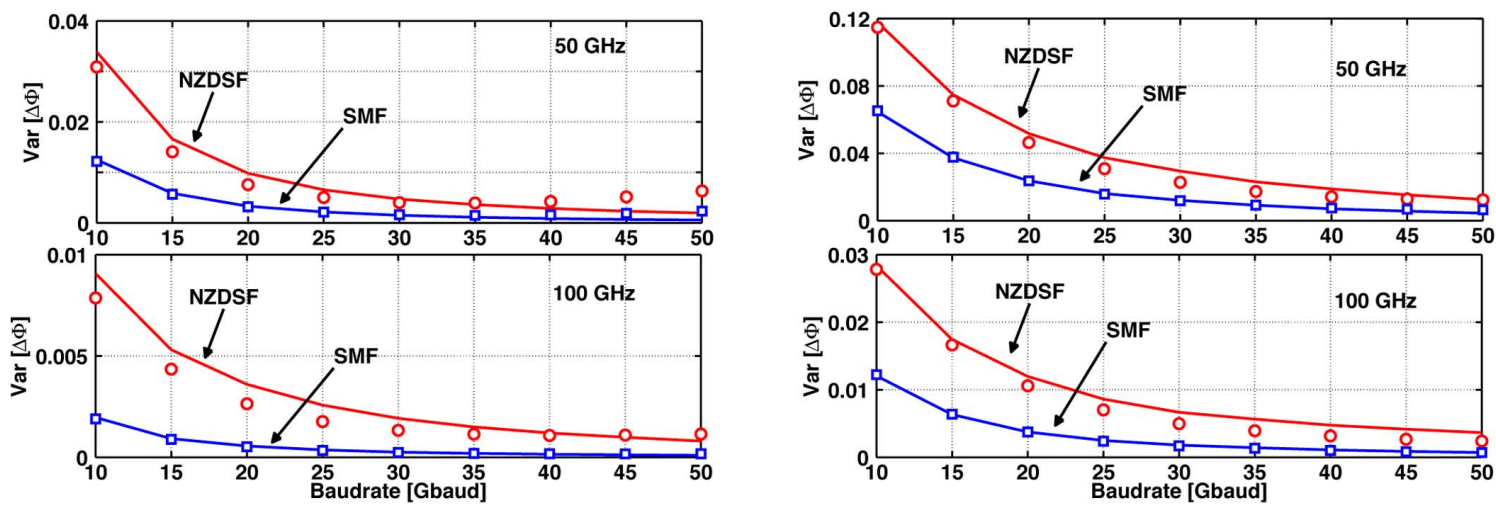

Fig. 6. XPM induced phase variance on DQPSK (left) or coherent QPSK ( $K=5$, right) reference channel at $P_{\text {in }}=2 \mathrm{dBm}$ versus QPSK channel baudrate. Same WDM 15 -span DM system as before, with $D_{\text {in }}=100 \mathrm{ps} / \mathrm{nm}, D_{\text {pre }}=\mathrm{SLR}, D_{\text {tot }}=0$, channel spacing $50 \mathrm{GHz}$ (top) and $100 \mathrm{GHz}$ (bottom), while the transmission fiber is either NZDSF or SMF. SSFM simulations without SPM (symbols) and XPM filter predictions (7) (solid).

nonlinear phase $\phi_{\mathrm{NL}}=0.22 \mathrm{rad}[30]$. SSFM simulations in this case did not include SPM. Hence, the predictions of the model are very close to simulation in all the tested scenarios. Note that the slight dependence of $\operatorname{Var}[\Delta \phi]$ on the baudrate is only caused by the random fluctuations of the Monte Carlo estimates of the PSD $C_{\mathrm{OOK}}(f)$ due to the finite bit-sequence. Replacing it with its theoretical limit (6) would yield flat curves at every power level, since $R_{S}$ doesn't appear in $H_{\mathrm{XPM}, p}(f)$ and $C_{\mathrm{OOK}}(f)$ is independent of the baudrate of the reference QPSK channel.

Fig. 6 shows instead the estimated phase variance after the differential filter versus QPSK channel baudrate, for both DQPSK reception (left graph), and coherent QPSK reception with $K=5$ (right graph). Solid lines refer to theory (7), while symbols to SSFM simulations. We also tested the same 15-span DM system at a lower $P_{\text {in }}=0 \mathrm{dBm}$, and the results (not reported) were qualitatively similar but with a much better fit.

By comparison with Fig. 5, we now note a strong dependence of $\operatorname{Var}[\Delta \phi]$ on the baudrate, which is clearly due to the action of the differential filter. This effect was already observed in [13], but ascribed to the walkoff effect. Even in this case the predictions of the theory (7) are rather close to the SSFM simulations when SPM is neglected.

\section{B. SP estimation}

Having numerically verified the effectiveness of the filterbased approach, we now turn to testing the SP approximations in (2) and (4).

One limit of the "Blachman" formula (1) is that the two noisy fields at times $t$ and $t-T$ are assumed to be independent, while for increasing walkoff the correlation time of the phase process $\Delta \phi(t)$ can be much longer than the symbol time $T_{s}$, thus clearly violating the independence assumption. Such correlations, by our experience, usually increase the penalty beyond the value predicted by (1). Another expected source of discrepancy with the actual penalty is the Gaussian assumption for the statistics of $\Delta \phi(t)$, which typically also leads to an over-estimation of penalty. Also, the analytical fits (2), (4) yield a penalty that slightly exceeds the predictions of the "Blachman" formulas (1), (3) at large XPM variance. Hence we expect the model to overestimate the penalty with respect to simulations.

To verify the match of the analytical formulas (2), (4) evaluated with the analytical variance (7) against the SSFM simulated
SP, we analyzed the same 15-span DM transmission systems already tested for the numerical results on phase variance. The SP was computed at BER $=10^{-5}$ using the fast semi-analytical Karhunen-Love (KL) method for DQPSK [36], while in the case of coherent QPSK with feedforward phase estimation, in the absence of a reliable semi-analytical BER estimation method, we computed the SP at BER $=10^{-3}$ using direct Monte Carlo error counting. We repeated the simulations 10 times, by varying the OOK random patterns and the delay between the channels. Again, $P_{\text {in }}$ was $2 \mathrm{dBm}$. Fig. 7 shows the obtained curves, where again solid lines denote theory (2), (4), symbols are simulations without SPM, while dashed lines are the "true" simulated SP including SPM.

From the figure we see that the theoretical fits (2), (4) give a reasonable match with simulations without SPM for SP values up to $\sim 2 \mathrm{~dB}$, but can lead to large overestimates at higher penalties (top figures). We verified that when the theoretical SP diverges form the simulated one, it is mainly due to the fact that the XPM-filter fails to give an exact prediction of the phase variance. In fact, substitution of the SSFM calculated phase variance in (2), (4) yields a more precise estimation of the SP, especially at higher baudrates.

When including SPM (dashed lines) the SP penalty increases because the SPM-induced (D)QPSK phase distortion causes an increase of the phase variance at higher baudrates. Such effect is not included in the theoretical model. If the channels are spaced by $50 \mathrm{GHz}$, the effect of XPM is stronger and thus the theoretical prediction is more accurate, at least up to about 30 Gbaud. At higher baudrates, the XPM is largely suppressed while the effect of SPM, which increases with the baudrate, gets more and more dominant. When the channels are spaced at $100 \mathrm{GHz}$, the XPM penalty is reduced and our approximation is less precise also at lower baudrates. Finally note that for DQPSK there is a minimum of the penalty around 30 Gbaud, while for coherent QPSK (which we showed to be more vulnerable to the XPM induced distortion) such a minimum is still not reached within the shown range of baudrates.

\section{CONCLUSIONS}

In this work we have provided a theoretical model able to explain the detailed mechanism of the XPM-induced performance degradation by neighboring $10 \mathrm{~Gb} / \mathrm{s}$ OOK channels on both incoherent DQPSK and coherent QPSK with feedforward phase 

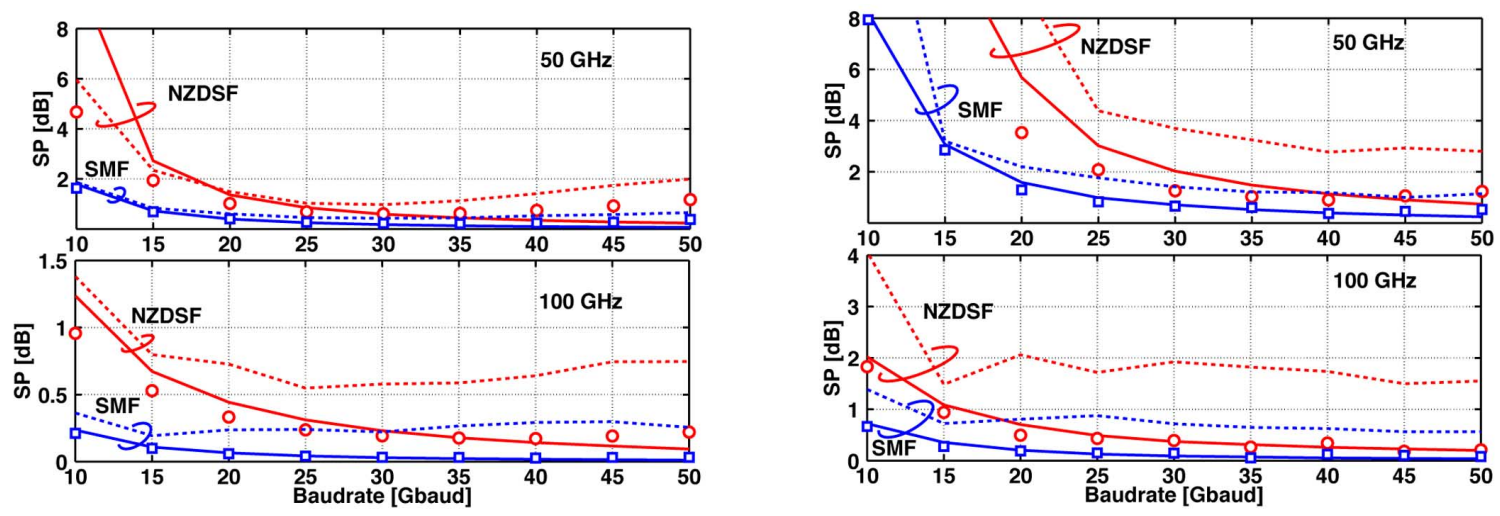

Fig. 7. XPM induced SP on DQPSK (left graphs) and coherent QPSK ( $K=5$, right graphs) reference channel for a 5-channel 15 -span hybrid DM system with $D_{\text {in }}=100 \mathrm{ps} / \mathrm{nm}, D_{\text {pre }}=\mathrm{SLR}, D_{\text {tot }}=0$ and average input power $P_{\text {in }}=2 \mathrm{dBm} / \mathrm{ch}$. The channel spacing is $50 \mathrm{GHz}$ (top) or $100 \mathrm{GHz}$ (bottom), while the fiber is either NZDSF or SMF. Theoretical SP (2), (4) (solid); simulated SP without SPM (symbols); simulated SP with SPM (dashed)

estimation. We have: i) derived IM-XPM filters, similar to the IM-IM filters derived in the past to study XPM penalty in OOK channels; ii) proved, by check against simulations when only XPM is active, that all that matters for the sensitivity penalty evaluation is the XPM variance induced on the test channel by OOK channels; iii) provided novel SP formulas for both incoherent DQPSK and coherent QPSK; iv) explained the reason why XPM-induced SP decreases with the baudrate of the phase modulated channel.

We traced back the origin mostly to the action of the generalized differential filter $H_{D}(f)$, which is present both in DQPSK and in feedforward phase-estimated coherent QPSK. The differential filter suppresses the low-frequency portion of XPM, more effectively when the baud-rate of the phase modulated channel is larger than the bit rate of the $10 \mathrm{G}$ OOK channels.

Such an analysis also explains why coherent QPSK, when the feedforward phase estimation is performed on more than one symbols, is more impaired by XPM than DQPSK at the same baudrate. Note that, although our analysis is performed for simplicity in a single-polarization setting, still one can infer results on performance of polarization division multiplexed (PDM) coherent QPSK by using the effective baud-rate of the QPSK channel. We therefore predict that at $40 \mathrm{~Gb} / \mathrm{s}$ coherent QPSK channels with polarization division multiplexing (10 Gbaud) would be even more impaired by $10 \mathrm{G}$ OOK channels. DQPSK (20 Gbaud) would be a better solution, but still would require some guard-band from 10G OOK channels to avoid any impact on the maximum achievable reach, especially over NZDSF. On the other hand, at $100 \mathrm{~Gb} / \mathrm{s}$, PDM-QPSK (25 Gbaud) would both allow the design of WDM systems with $50 \mathrm{GHz}$ spacing and would be less impaired by neighboring channels thanks to the increased baudrate.

Finally note that effects such as nonlinear polarization rotation and its interplay with PMD are not included in the analysis and should be investigated before a final complete picture of the performance degradation due to XPM is obtained.

\section{APPENDIX I}

\section{ON THE GAUSSIAN APPROXIMATION OF XPM}

In this Appendix we wish to justify the Gaussian approximation for the XPM.
For each OOK pump at spectral distance $\Delta \lambda$ from probe, the number of (independent) symbols that predominantly contribute to the induced XPM at a specific sampling time on the probe is related to both the uncompensated per-span walkoff $T_{W \text {,span }}=D_{T X} \Delta \lambda L_{\text {eff }} / T_{\mathrm{OOK}}$ accumulated by the pump over one effective length $L_{\text {eff }} \cong(1 / \alpha)$ along the transmission fiber [10], normalized by the OOK symbol time $T_{\mathrm{OOK}}$, and to the (normalized) per-span average walkoff after in-line compensation $T_{W \text {,avg }}=D_{\text {in }} \Delta \lambda / T_{\mathrm{OOK}}$, where $D_{\text {in }}[\mathrm{ps} / \mathrm{nm}]$ is the in-line dispersion per span. A little thought about the sawtooth sliding movement of the pump channel relative to the probe channel reveals that the number of interfering bits for each pump for time-asynchronous WDM channels over $N$ spans is

$$
N_{\text {int }}=1+\left\lceil(N-1) T_{W, \text { avg }}+T_{W, \text { span }}\right\rceil
$$

For zero walkoff, $N_{\text {int }}=1$ since only one pump bit generates XPM on the probe at a specific sampling time. For instance, for a 15 span DM system with $D_{\text {in }}=100 \mathrm{ps} / \mathrm{nm} / \mathrm{span}$ as in Figs. 6 and 7, the 2 nearest pumps at a channel spacing of $50 \mathrm{GHz}$ both have $N_{\text {int }}=8$ for SMF fiber and $N_{\text {int }}=7$ for NZDSF fiber (fiber data in Section V). Thus the dominant XPM due to the 2 nearest pumps is the sum of about 16 terms for SMF, and of 14 terms for NZDSF, which are already sufficient to provide a reasonable Gaussian fit of the XPM PDF in the modal range. However the XPM PDF certainly has limited tails, while the Gaussian tail is unbounded. So the question is: how much does the tail error impact the BER formula? The answer is that when the ASE induced phase noise is (much) larger than the XPM, then the exact tail shape of XPM is less important. The ASE-induced phase has variance for differential detection equal to [17] $\sigma_{\text {ase }}^{2}=2 *(1 / 2 \rho)=1 / \rho\left[\mathrm{rad}^{2}\right]$. For DQPSK reception at $\mathrm{BER}=10^{-5}$ one has $\rho=31.4$ hence $\sigma_{\text {ase }}^{2}=0.032 \mathrm{rad}^{2}$. If one checks the XPM variance values in Fig. 6, one sees that in the worst-case of NZDSF fiber, $50 \mathrm{GHz}$ spacing, at a DQPSK baudrate of $10 \mathrm{Gbaud}$ one has $\sigma_{\mathrm{XPM}}^{2}=0.03 \mathrm{rad}^{2}$ i.e., equal to the ASE variance, hence here the Gaussian assumption for XPM leads to some over-estimation of the SP. But already at a baudrate of 20 Gbaud the variance has dropped to 0.01 , i.e., we are adding ASE phase noise to an XPM whose variance is $1 / 3$ of that of ASE: hence the dominant error events are due to typical values of XPM (in the modal range of the XPM PDF 
which looks Gaussian) added to large deviations of ASE phase noise (i.e., tail values).

That's the main reason why the complete tail distribution of the XPM does not actually affect much the SP that we infer from "Blachman's" formulae (1)-(3) with Gaussian XPM.

\section{APPENDIX II}

\section{PSK BER WITH GAUSSIAN PHASE NOISE}

This Appendix provides a summary of BER results for M-ary differential and coherent PSK. Although most of these results can be found, e.g., in the book by Ho [16], we find it useful to present them to the reader in a general and self-contained form.

We first consider the case of differential (or "incoherent") reception of an M-ary DPSK signal. Let $\Delta \varphi=\varphi_{t}-\varphi_{T}+\Delta \phi$ be the angle between the two noisy received complex optical fields $E(t)=A(t)+m(t)$ and $E(t-T)=A(t-T)+m(t-T)$ that get combined in the receiver interferometer, where $\varphi_{t}$ is the angle between $E(t)$ and $A(t)$ produced by the additive ASE optical noise $m(t),{ }^{2}$ while $\varphi_{T}$ is the angle between $E(t-T)$ and $A(t-T)$, and $\Delta \phi$ is the phase angle (or "offset") between the signal samples $A(t)$ and $A(t-T)$.

Since the detection problem is symmetric, we assume symbol " 0 " is transmitted, so that the received fields $E(t)$ and $E(t-T)$ should ideally be aligned on the positive real axis. An error occurs in the detection process if the differential angle $\Delta \varphi$ falls outside the range $[-\pi / M, \pi / M]$. Hence the probability of a symbol error in MDPSK is

$$
P_{e}(M)=1-\int_{-\pi / M}^{\pi / M} f_{\Delta \varphi}(x) d x
$$

where $f_{\Delta \varphi}(x)$ is the probability density function of the random variable (RV) $\Delta \varphi$. We expand the PDF $f_{\Delta \varphi}(x)$ over its nonzero support $x \in[-\pi, \pi]$ in a Fourier series

$$
\begin{aligned}
f_{\Delta \varphi}(x) & =\frac{1}{2 \pi} \sum_{n=-\infty}^{\infty} \Psi_{\Delta \varphi}(n) e^{-j n x} \\
& =\frac{1}{2 \pi}+\frac{1}{\pi} \sum_{n=1}^{\infty} \operatorname{Re}\left[\Psi_{\Delta \varphi}(n) e^{-j n x}\right]
\end{aligned}
$$

where $\Psi_{\Delta \varphi}(n) \triangleq \mathrm{E}\left[e^{j n \Delta \varphi}\right]$ is the characteristic function $(\mathrm{CF})$ of $\Delta \varphi$, which has Hermitian symmetry since $f_{\Delta \varphi}(x)$ is real. Assume now independence between the RV's $\varphi_{t}$ and $\varphi_{T}$ having PDFs $f_{\varphi_{t}}$ and $f_{\varphi_{T}}$, respectively. Then, assuming the offset $\Delta \phi$ is known, we get the CF of the phase difference $\mathrm{RV}$ as

$$
\Psi_{\Delta \varphi}(n)=e^{j n \Delta \phi} \underbrace{\mathrm{E}\left[e^{j n \varphi_{t}}\right]}_{\Psi_{\varphi_{t}}(n)} \underbrace{\mathrm{E}\left[e^{-j n \varphi_{T}}\right]}_{\Psi_{\varphi_{T}^{*}}^{*}(n)} .
$$

${ }^{2}$ We consider here only the ASE co-polarized with the signal. The extension to the case of both ASE polarizations is simple [16] and does not significantly change the numerical result
Now, use (12), (11) into (10) to get a general expression of $P_{e}(M)$

$$
\begin{aligned}
P_{e}(M)=\left(1-\frac{1}{M}\right)-\frac{2}{\pi} \sum_{n=1}^{\infty}\left|\Psi_{\varphi_{t}}(n)\right|\left|\Psi_{\varphi_{T}}(n)\right| \\
\quad \cdot \frac{\sin \left(n \frac{\pi}{M}\right)}{n} \cos \left(a_{t}(n)-a_{T}(n)+n \Delta \phi\right)
\end{aligned}
$$

where $a_{t}(n)$ is the phase of the complex $\Psi_{\varphi_{t}}(n)$, and $a_{T}(n)$ is similarly defined.

Now, when $\tilde{m(t)}=m_{p t}+j m_{q t}$ is a circular Gaussian vector, with $m_{p t}$ and $m_{q t}$ zero-mean independent Gaussian RV's with common variance $\sigma_{0}^{2}$, one can prove that the angle $\varphi_{t}$ has a Bennet PDF whose CF can be written explicitly as the following real quantity [14]:

$$
\Psi_{\varphi_{t}}(n)=\frac{\sqrt{\pi}}{2} \sqrt{\rho_{t}} e^{-\frac{\rho_{t}}{2}}\left[I_{\frac{n-1}{2}}\left(\frac{\rho_{t}}{2}\right)+I_{\frac{n+1}{2}}\left(\frac{\rho_{t}}{2}\right)\right]
$$

where $\rho_{t}=A(t)^{2} / 2 \sigma_{0}^{2}$ is the instantaneous SNR for the sample at time $t$. Therefore, when the noises $\tilde{m}(t)$ and $\tilde{m}(t-T)$ are independent circular Gaussian RV's with identical variance, one can similarly define the SNR $\rho_{T}=A(t-T)^{2} / 2 \sigma_{0}^{2}$ at time $t-T$, and using (14) the MDPSK error probability formula (13) becomes

$$
\begin{aligned}
P_{e}(M)= & \left(1-\frac{1}{M}\right)-\frac{1}{2} \sqrt{\rho_{t} \rho_{T}} e^{-\frac{\rho_{t}}{2}-\frac{\rho_{T}}{2}} \\
& \cdot \sum_{n=1}^{\infty} \frac{\sin \left(n \frac{\pi}{M}\right)}{n} \cdot \cos (n \Delta \phi) \\
& \cdot\left[I_{\frac{n-1}{2}}\left(\frac{\rho_{t}}{2}\right)+I_{\frac{n+1}{2}}\left(\frac{\rho_{t}}{2}\right)\right] \\
& \times\left[I_{\frac{n-1}{2}}\left(\frac{\rho_{T}}{2}\right)+I_{\frac{n+1}{2}}\left(\frac{\rho_{T}}{2}\right)\right] .
\end{aligned}
$$

In coherent communications, the performance can be evaluated as for differential MPSK by considering that in this case the second beating field $\tilde{E}(t-T) \equiv \tilde{A}(t-T)$ comes from the local oscillator and is thus not affected by ASE. Hence the angle between the two noisy received complex signal fields is $\Delta \varphi=\varphi_{t}+\Delta \phi$. Since $\varphi_{T} \equiv 0$ is deterministic, the error probability formula (13) still applies. Thus using $\Psi_{\varphi_{T}}(n) \equiv 1$ and (14) into (13) we get for coherent MPSK reception

$$
\begin{array}{r}
P_{e}(M)=\left(1-\frac{1}{M}\right)-\frac{2}{\pi} \frac{\sqrt{\pi}}{2} \sqrt{\rho_{0}} e^{-\frac{\rho_{0}}{2}} \cdot \sum_{n=1}^{\infty} \frac{\sin \left(n \frac{\pi}{M}\right)}{n} \\
\cdot \cos (n \Delta \phi)\left[I_{\frac{n-1}{2}}\left(\frac{\rho_{0}}{2}\right)+I_{\frac{n+1}{2}}\left(\frac{\rho_{0}}{2}\right)\right] .
\end{array}
$$

Finally, when $\Delta \phi$ is Gaussian distributed, averaging (15) and (16) with respect to $\Delta \phi$ and using the fact that $\mathrm{E}[\cos (n \Delta \phi)]=$ $\mathrm{E}\left[e^{j n \Delta \phi}\right]=e^{-(\operatorname{Var}[\Delta \phi] / 2) n^{2}}$, we obtain, as done by Nicholson for DPSK [37], the desired expression of the BER, which in (1) and (3) is specialized to DQPSK/QPSK respectively $(M=4)$ by considering that the BER is approximately half the symbol error probability [38, footnote p. 1834]. 


\section{APPENDIX III \\ IM-XPM FILTER}

Consider the $k$ th span of length $\ell_{k}$ of an $N$-span DM optical link. At the DM link input consider a CW input probe signal at wavelength $\lambda_{s}$, having a small enough power to neglect self-phase modulation effects, along with an on-off keying (OOK) modulated pump signal at wavelength $\lambda_{p}$ having power whose Fourier transform is $P_{p}(0, \omega) e^{-j\left(\omega^{2} / 2\right) \beta_{\text {pre }} \text {, where }}$ $\beta_{\text {pre }}=\beta_{2, \text { pre }} \ell_{\text {pre }}\left[\mathrm{ps}^{2}\right]$ is the GVD cumulated in the link pre-compensation fiber of length $\ell_{\text {pre }}$, and $P_{p}(0, \omega)$ is the input pump Fourier transform.

Following the same reasoning as in [20]-[22], the pump power at coordinate $z$ within the $k$ th span in the retarded time frame of the signal, using the GVD induced IM-IM small signal conversion [39], has Fourier transform

$$
\begin{aligned}
P_{p}(z, \omega)=P_{p}(0, \omega) e^{j \omega d_{a}(k)} & e^{\left[-\alpha+j \omega d_{s p, k}\right] z} \\
\cdot & \cos \left[\frac{\omega^{2}}{2}\left(\beta_{a}(k)+\beta_{2, k} z\right)\right]
\end{aligned}
$$

due to walkoff ( $d_{s p, k} \cong D_{k} \Delta \lambda_{s p}$ is the walkoff parameter, being $D_{k}$ the fiber dispersion and $\Delta \lambda_{s p}=\lambda_{s}-\lambda_{p}$ the channel spacing), fiber attenuation parameter $\alpha$, and GVD parameter $\beta_{2, k}$. Here $\beta_{a}(k)=\beta_{\text {pre }}+\sum_{i=1}^{k-1} \beta_{s, j}$ is the dispersion accumulated from system input (including pre-compensation) until the span input, being $\beta_{s, i}$ the accumulated GVD $\left[p s^{2}\right]$ in the $i$ th span after in-line compensation, while $d_{a}(k)=d_{s p, p r e} \ell_{\text {pre }}+\sum_{i=1}^{k-1} d_{a, i}$ is the accumulated walkoff from system input to span input, being $d_{a, i}$ the accumulated walkoff in the $i$ th span after in-line compensation. In writing (17) we are also assuming for simplicity that: i) all spans have the same input power, i.e., the in-line amplifiers recover all span losses; ii) the in-line dispersion compensating fibers are purely linear devices. The probe phase induced at $z$ through XPM by propagation of such a pump over an infinitesimal segment $d z$ has Fourier transform $d \Theta(z, \omega)=-2 \gamma P_{p}(z, \omega) d z$. Such a phase modulation enters, if the nonlinear effects of the remaining line segment are neglected, an equivalent pure-GVD trunk composed of the remaining $\ell_{k}-z \mathrm{~km}$ of fiber within the kth span, followed by the remaining spans to the DM system end. Such a purely linear trunk produces by PM-PM GVD conversion at the DM system output an infinitesimal output XPM of [21], [39]: $d \Theta_{\mathrm{XPM}}(\omega)=\cos \left(\left(\omega^{2} / 2\right)\left(\beta_{r}-\beta_{a}(k)-\beta_{2, k} z\right) d \Theta(z, \omega)\right.$, where $\beta_{r}\left[p s^{2}\right]$ is the residual (i.e., total) dispersion accumulated over the entire DM link (which does include also a post-compensating fiber when present), hence $\left(\beta_{r}-\beta_{a}(k)-\beta_{2, k} z\right)$ is the dispersion cumulated from the local coordinate $z$ within span $k$ until the DM system end. Integrating over all infinitesimal contributions from $z=0$ to $z=\ell_{k}$ on span $k$ one finally gets the output cross phase due to span $k$ as: $\Theta_{\mathrm{XPM}}^{(k)}(\omega)=\int_{0}^{\ell_{k}} d \Theta_{\mathrm{XPM}}(\omega)=H_{\mathrm{XPM}}^{(k)}(\omega) P_{p}(0, \omega)$, where the $k$ th span IM-XPM filter is

$$
\begin{gathered}
H_{\mathrm{XPM}}^{(k)}(\omega)=-2 \gamma e^{j \omega d_{a}(k)} \int_{0}^{\ell_{k}} \cos \left[\frac{\omega^{2}}{2}\left(\beta_{a}(k)+\beta_{2, k} z\right)\right] \\
\cdot \cos \left(\frac{\omega^{2}}{2}\left(\beta_{r}-\beta_{a}(k)-\beta_{2, k} z\right)\right) e^{\left(-\alpha+j \omega d_{s p, k}\right) z} d z .
\end{gathered}
$$

The total output XPM on probe channel in this linearized model is the sum of the contributions of all $N$ spans: $\Theta_{\mathrm{XPM}}(\omega)=\sum_{k=1}^{N} H_{\mathrm{XPM}}^{(k)}(\omega) P_{p}(0, \omega)$. Solving the integral in (18) gives the explicit global IM-XPM filter:

$$
\begin{aligned}
H_{\mathrm{XPM}}(\omega) & \\
= & -2 \gamma \sum_{k=1}^{N} e^{j \omega d_{a}(k)} \frac{1}{4} \\
& \cdot\left\{e^{j\left(\beta_{r}-2 \beta_{a}(k)\right) \frac{\omega^{2}}{2}} \frac{1-e^{-\left(\alpha+j\left(\omega^{2} \beta_{2, k}-\omega d_{s p, k}\right)\right) \ell_{k}}}{\alpha+j\left(\omega^{2} \beta_{2, k}-\omega d_{s p, k}\right)}\right. \\
& +e^{-j\left(\beta_{r}-2 \beta_{a}(k)\right) \frac{\omega^{2}}{2}} \frac{1-e^{-\left(\alpha-j\left(\omega^{2} \beta_{2, k}+\omega d_{s p, k}\right)\right) \ell_{k}}}{\alpha-j\left(\omega^{2} \beta_{2, k}+\omega d_{s p, k}\right)} \\
& \left.+2 \cos \left(\beta_{r} \frac{\omega^{2}}{2}\right) \frac{1-e^{-\left(\alpha-j \omega d_{s p, k}\right) \ell_{k}}}{\alpha-j \omega d_{s p, k}}\right\} .
\end{aligned}
$$

If we assume long spans $\left(\ell_{k} \gg 1 / \alpha\right)$, a uniform channel spacing so that $\lambda_{s}-\lambda_{p}=(s-p) \Delta \lambda$, with $\Delta \lambda=\left(\lambda^{2} / 2 \pi c\right) \Delta \omega>0$, and assume that our reference channel is $s=0$, then (19) becomes

$$
\begin{aligned}
& H_{\mathrm{XPM}}(\omega) \\
& =-\frac{\gamma}{2 \alpha} e^{j \omega p \beta_{\mathrm{pre}} \Delta \omega} \\
& \cdot\left\{\frac{e^{j \frac{\omega^{2}}{2}\left(\beta_{r}-2 \beta_{\mathrm{pre}}\right)} \sum_{k=1}^{N} e^{-j[\omega-p \Delta \omega] \omega \beta_{s}(k-1)}}{1+j \frac{\beta_{2}}{\alpha} \omega(\omega-p \Delta \omega)}\right. \\
& +\frac{e^{-j \frac{\omega^{2}}{2}\left(\beta_{r}-2 \beta_{\text {pre }}\right)} \sum_{k=1}^{N} e^{j[\omega+p \Delta \omega] \omega \beta_{s}(k-1)}}{1-j \frac{\beta_{2}}{\alpha} \omega(\omega+p \Delta \omega)} \\
& \left.+\frac{\left(e^{j \frac{\omega^{2}}{2} \beta_{r}}+e^{-j \frac{\omega^{2}}{2} \beta_{r}}\right) \sum_{k=1}^{N} e^{j \omega p \beta_{s} \Delta \omega(k-1)}}{1-j \frac{\beta_{2}}{\alpha} \omega p \Delta \omega}\right\} .
\end{aligned}
$$

Define now $x \triangleq e^{j W \beta_{s}}$, and introduce the following interference term

$$
I_{p}(W) \triangleq \frac{1}{N} \sum_{k=0}^{N-1} x^{k}=e^{j W \beta_{s} \frac{N-1}{2}} \frac{\sin \left(W \frac{\beta_{s} N}{2}\right)}{N \sin \left(W \frac{\beta_{s}}{2}\right)} .
$$

Also define a walkoff term as

$$
H_{W p}(W)=\frac{1}{1-j \frac{\beta_{2}}{\alpha} W}
$$

and finally define the following filter:

$$
\begin{array}{r}
H_{p}(\omega) \triangleq e^{j \omega p \Delta \omega \beta_{\mathrm{pre}}} I_{p}(\omega p \Delta \omega) H_{W p}(\omega p \Delta \omega)+e^{j \omega(\omega+p \Delta \omega) \beta_{\mathrm{pre}}} \\
\quad \times I_{p}(\omega(\omega+p \Delta \omega)) \cdot H_{W p}(\omega(\omega+p \Delta \omega)) \cdot
\end{array}
$$

It is now easy to see that, using the new filter (23), the IM-XPM filter in (20) can finally be expressed as in (5), with $\Phi_{N L}=N(\gamma / \alpha) P_{\text {in }, \mathrm{p}}$.

\section{ACKNOWLEDGMENT}

The authors would like to acknowledge the stimulating interactions with S. Bigo and A. Chiarotto of Alcatel-Lucent. 


\section{REFERENCES}

[1] A. Gnauck, G. Charlet, P. Tran, P. Winzer, C. Doerr, J. Centanni, E. Burrows, T. Kawanishi, T. Sakamoto, and K. Higuma, "25.6-Tb/s WDM transmission of polarization-multiplexed RZ-DQPSK signals," J. Lightw. Technol., vol. 26, no. 1, pp. 79-84, Jan. 2008.

[2] H. Sun, K.-T. Wu, and K. Roberts, "Real-time measurements of a 40 Gb/s coherent system," Opt. Exp., vol. 16, no. 2, pp. 873-879, 2008.

[3] J. Renaudier, G. Charlet, M. Salsi, O. B. Pardo, H. Mardoyan, P. Tran, and S. Bigo, "Linear fiber impairments mitigation of 40-Gbit/s polarization-multiplexed QPSK by digital processing in a coherent receiver," J. Lightw. Technol., vol. 26, no. 1, pp. 36-42, Jan. 2008.

[4] C. Fürst et al., " $43 \mathrm{~Gb} / \mathrm{s}$ RZ-DQPSK DWDM field trial over $1047 \mathrm{~km}$ with mixed $43 \mathrm{~Gb} / \mathrm{s}$ and $10.7 \mathrm{~Gb} / \mathrm{s}$ channels at 50 and $100 \mathrm{GHz}$ channel spacing," presented at the Proc. ECOC 2006, 2006, PDP Th4.1.4, unpublished.

[5] H. Griesser et al., " $43 \mathrm{~Gb} / \mathrm{s} \mathrm{RZ-DQPSK} \mathrm{transmission} \mathrm{over} \mathrm{a} 660 \mathrm{~km}$ $10.7 \mathrm{~Gb} / \mathrm{s}$ DWDM link," presented at the Proc. ECOC 2006, 2006, paper Th1.6.6, unpublished.

[6] S. Chandrasekhar and X. Liu, "Impact of channel plan and dispersion map on hybrid DWDM transmission of $42.7-\mathrm{Gb} / \mathrm{s}$ DQPSK and 10.7-Gb/s OOK on 50-GHz grid," IEEE Photon. Technol. Lett., vol. 19, no. 22, pp. 1801-1803, Nov. 2007.

[7] S. Bigo, G. Charlet, O. Bertrand Pardo, and J. Renaudier, "Characterization of the impact of non-linear effects in coherent transmission experiments," in Digest of the IEEE/LEOS Summer Topical Meetings 2008, July 2008, pp. 125-126.

[8] O. Bertran-Pardo, J. Renaudier, G. Charlet, H. Mardoyan, P. Tran, and S. Bigo, "Nonlinearity limitations when mixing 40-Gb/s coherent PDM-QPSK channels with preexisting 10-Gb/s NRZ channels," IEEE Photon. Technol. Lett., vol. 20, no. 15, pp. 1314-1316, Aug. 2008.

[9] H. Griesser and J. Elbers, "Influence of cross-phase modulation induced nonlinear phase noise on DQPSK signals from neighbouring OOK channels," presented at the Proc. ECOC 2005, 2005, paper Tu1. 2.2, unpublished.

[10] K.-P. Ho, "Error probability of DPSK signals with cross-phase modulation induced nonlinear phase noise," IEEE J. Sel. Topics Quantum Electron., vol. 10, no. 2, pp. 421-427, March-pril 2004.

[11] R. Luis, B. Clouet, A. Teixeira, and P. Monteiro, "Analytical modeling of the cross-phase modulation-induced degradation in mixed DPSK and ASK transmission systems," presented at the Proc. ICTON 2007, 2007, paper Mo.P.17, unpublished.

[12] R. Luis, B. Clouet, A. Teixeira, and P. Monteiro, "Pump-probe analysis of the cross-phase modulation degradation induced by $10 \mathrm{Gbit} / \mathrm{s}$ amplitude-shift-keyed signals on $40 \mathrm{Gbit} / \mathrm{s}$ DPSK signals," Opt. Lett., vol. 32 , no. 19 , p. 2786 , Oct. 2007.

[13] O. Vassilieva, T. Hoshida, J. C. Rasmussen, and T. Naito, "Symbol rate dependency of XPM-induced phase noise penalty on QPSK-based modulation formats," presented at the Proc. ECOC 2008, 2008, paper We.1.E.4, unpublished.

[14] N. Blachman, "The effect of phase error on DPSK error probability," IEEE Trans. Commun., vol. 29, no. 3, pp. 364-365, Mar. 1981.

[15] K.-P. Ho, "The effect of interferometer phase error on direct-detection DPSK and DQPSK signals," IEEE Photon. Technol. Lett., vol. 16, no. 1, pp. 308-310, Jan. 2004.

[16] K.-P. Ho, Phase-Modulated Optical Communication Systems. New York: Springer, 2005.

[17] J. Gordon and L. Mollenauer, "Phase noise in photonic communications systems using linear amplifiers," Opt. Lett, vol. 15, no. 23, pp. 1351-1353, 1990.

[18] T.-K. Chiang, N. Kagi, M. Marhic, and L. Kazovsky, "Cross-phase modulation in fiber links with multiple optical amplifiers and dispersion compensators," J. Lightw. Technol., vol. 14, no. 3, pp. 249-260, Mar. 1996.

[19] A. Bononi, C. Francia, and G. Bellotti, "Impulse response of crossphase modulation filters in multi-span transmission systems with dispersion compensation," Opt. Fiber Technol., vol. 4, no. 4, pp. 371-383, 1998.

[20] M. Varani, G. Bellotti, A. Bononi, and C. Francia, "Analysis of cross-phase modulation induced intensity noise in high-speed dispersion compensated transmission systems," presented at the Proc. LEOS '98, 1998, paper WBB4, unpublished.

[21] A. Bononi, G. Bellotti, M. Varani, and C. Francia, "SPM/XPM-induced intensity distortion in WDM systems," in Optical Networking, A. Bononi, Ed. London, U.K.: Springer-Verlag, 1999, pp. 383-398.
[22] A. Cartaxo, "Cross-phase modulation in intensity modulation-direct detection WDM systems with multiple optical amplifiers and dispersion compensators," J. Lightw. Technol., vol. 17, no. 2, pp. 178-190, Feb. 1999.

[23] R. Hui, Y. Wang, K. Demarest, and C. Allen, "Frequency response of cross-phase modulation in multispan WDM optical fiber systems," IEEE Photon. Technol. Lett., vol. 10, no. 9, pp. 1271-1273, Sep. 1998.

[24] A. Cartaxo, "Impact of modulation frequency on cross-phase modulation effect in intensity modulation-direct detection WDM systems," IEEE Photon. Technol. Lett., vol. 10, no. 9, pp. 1268-1270, Sep. 1998.

[25] G. Bellotti, M. Varani, C. Francia, and A. Bononi, "Intensity distortion induced by cross-phase modulation and chromatic dispersion in optical-fiber transmissions with dispersion compensation," IEEE Photon. Technol. Lett., vol. 10, no. 12, pp. 1745-1747, Dec. 1998.

[26] R. Killey, H. Thiele, V. Mikhailov, and P. Bayvel, "Prediction of transmission penalties due to cross-phase modulation in WDM systems using a simplified technique," IEEE Photon. Technol. Lett., vol. 12, no. 7, pp. 804-806, Jul. 2000.

[27] A. Bononi, P. Serena, and M. Bertolini, "Unified analysis of weaklynonlinear dispersion-managed optical transmission systems from perturbative approach," C. R. Physique, vol. 9, pp. 947-962, 2008.

[28] R. Luis and A. Cartaxo, "Analytical characterization of SPM impact on XPM-induced degradation in dispersion-compensated WDM systems," J. Lightw. Technol., vol. 23, no. 3, pp. 1503-1513, 2005.

[29] Y. Frignac, J.-C. Antona, and S. Bigo, "Enhanced analytical engineering rule for fast optimization dispersion maps in $40 \mathrm{Gbit} / \mathrm{s}-$ based transmission," presented at the Proc. OFC 2004, 2008, Paper OTuN3, unpublished.

[30] A. Bononi, P. Serena, and A. Orlandini, "A unified design framework for single-channel dispersion-managed terrestrial systems," J. Lightw. Technol., vol. 26, no. 22, pp. 3617-3631, Nov. 2008

[31] E. Bedrosian and S. Rice, "Distortion and crosstalk of linearly filtered, angle modulated signals," Proc. IEEE, vol. 56, no. 5, pp. 2-13, Jan. 1968.

[32] A. J. Viterbi and A. M. Viterbi, "Nonlinear estimation of PSK-modulated carrier phase with application to burst digital transmission," IEEE Trans. Inf. Theory, vol. 29, no. 4, pp. 543-551, 1983.

[33] E. Ip and J. Kahn, "Feedforward carrier recovery for coherent optical communications," J. Lightw. Technol., vol. 25, no. 9, pp. 2675-2692, Sep. 2007.

[34] D.-S. Ly-Gagnon, S. Tsukamoto, K. Katoh, and K. Kikuchi, "Coherent detection of optical quadrature phase-shift keying signals with carrier phase estimation," J. Lightw. Technol., vol. 24, no. 1, pp. 12-21, Jan. 2006.

[35] M. Bertolini, P. Serena, N. Rossi, and A. Bononi, "Numerical Monte Carlo comparison between coherent PDM-QPSK/OOK and incoherent DQPSK/OOK hybrid systems," presented at the Proc. ECOC2008, 2008, paper P.4.15, unpublished.

[36] P. Serena, A. Orlandini, and A. Bononi, "Parametric-gain approach to the analysis of single-channel DPSK/DQPSK systems with nonlinear phase noise," J. Lightw. Technol., vol. 24, no. 5, pp. 2026-2037, May 2006.

[37] G. Nicholson, "Probability of error for optical heterodyne DPSK system with quantum phase noise," Electron. Lett., vol. 20, no. 24, pp. 1005-1007, 1984, 22.

[38] R. Pawula, S. Rice, and J. Roberts, "Distribution of the phase angle between two vectors perturbed by Gaussian noise," IEEE Trans. Commun., vol. 30, no. 8, pp. 1828-1841, Aug. 1982.

[39] J. Wang and K. Petermann, "Small signal analysis for dispersive optical fiber communication systems," J. Lightw. Technol., vol. 10, no. 1, pp. 96-100, Jan. 1992.

Alberto Bononi, Photograph and biography not available at the time of publication.

Marco Bertolini, Photograph and biography not available at the time of publication.

Paolo Serena, Photograph and biography not available at the time of publication.

Giovanni Bellotti, Photograph and biography not available at the time of publication. 